\title{
Clinical value of CT diagnosis in abdominal trauma.
}

\author{
Xiang Li*, Shu-Tong Zhang, Xiang Wang, Jing-Ting Chen \\ Department of Radiology, Wuhan Central Hospital, Affiliated to Tongji Medical College of Huazhong University of \\ Science and Technology, Wuhan, PR China
}

\begin{abstract}
Objective: This paper discusses the clinical value of CT diagnosis in abdominal trauma.

Method: Eighty patients with abdominal trauma admitted in our hospital from April 2015 to July 2017 were selected and subjected to B-ultrasonography and CT scan. Surgical and pathological diagnoses were used as the gold standard. The two diagnostic methods were compared in terms of sensitivity, specificity, accuracy, and diagnosis accordance rate of organ injury in the abdomen.

Results: Based on surgical and pathological diagnoses, 75 of 80 patients $(93.8 \%)$ were confirmed to have organ injury in the abdomen and included 25 patients with spleen damage, 13 patients with liver damage, 10 patients with kidney damage, 10 patients with pancreas damage, 9 patients with small intestine/duodenum damage, and 8 patients with peritoneum damage. The sensitivity, specificity, and accuracy of B-ultrasonography are $82.6 \%, 80.0 \%$, and $82.5 \%$, which are not significantly different from those of CT scan $(90.7 \%, 80.0 \%$, and $90.0 \%)(P>0.05)$. The diagnosis accordance rates of $B$ ultrasonography in the spleen, liver, kidney, pancreas, small intestine/duodenum, and peritoneum damages are $\mathbf{9 2 . 0} \%, \mathbf{9 2 . 3 \%}, \mathbf{1 0 0 . 0} \%, \mathbf{8 0 . 0} \%, \mathbf{7 7 . 8 \%}$, and $\mathbf{7 5 . 0 \%}$, respectively, and those of CT scan are $100.0 \%, 100.0 \%, 90.0 \%, 90.0 \%, 100.0 \%$, and $87.5 \%$, respectively; the difference between the two methods is not significant $(\mathbf{P}>0.05)$.

Conclusions: B-ultrasonography and CT scan present similar clinical results in abdominal trauma diagnosis. These procedures are noninvasive, simple to operate, and worthy of further clinical promotion and application.
\end{abstract}

Keywords: Abdominal trauma, B-ultrasonography, CT, Clinical value.

Accepted on October 26, 2017

\section{Introduction}

Abdominal trauma is a common traumatic injury and has high fatality rate. The incidence rate of abdominal trauma increases due to the frequent occurrence of traffic accidents [1]. The clinical symptoms of abdominal trauma mainly include nausea, emesis, stomach-ache, and hematuresis, which affect the daily life and work activities of patients [2]. Abdominal trauma is relatively complicated and is mainly determined by organ injury in the abdomen. Organ injuries in the abdomen can cause infection, hemorrhage, shock, peritonitis, and even death [3]. Hence, early diagnosis and interventions should be given to patients to protect their lives. In this study, 80 patients with abdominal trauma admitted in our hospital from April 2015 to July 2017 were selected for evaluation of the clinical value of CT diagnosis.

\section{Information and Method}

\section{General information}

Eighty patients with abdominal trauma admitted in our hospital from April 2015 to July 2017 were selected. The participants included 32 females and 48 males, aged 20 to 76 y (average of $43.4 \pm 9.7 \mathrm{y})$. The main causes of trauma among the patients were traffic accident $(n=50)$, occupational injury $(n=15)$, fall accident from a high place $(n=10)$, and fights $(n=5)$. The shortest time from the injury to the diagnosis is $2 \mathrm{~h}$, and the longest time is $24 \mathrm{~h}(8.1 \pm 2.4 \mathrm{~h}$ in average $)$.

\section{Methods}

B-ultrasonography: Patients were examined in supine, sitting, prostrate, and lateral positions by using real-time diasonograph under the probe frequency range of 4.0-6.0 $\mathrm{MHz}$. The organs at lesion and surrounding organs were examined carefully in terms of damage and pain. The abdominal cavity was also examined for the presence of seroperitoneum. Inspection of organs, such as the liver, kidney, and pancreas, was performed in different regions of the abdomen, noting for substantial organ damage. Abnormal representations in the ultrasonogram were used as the basis for diagnosis; these representations included abnormal organ shape parenchymatous anomaly, disturbed echo area, and enhanced echo. Hematoma was diagnosed by the existence of hypoecho or opaque dark area in 
fluid, and visceral laceration was diagnosed by interrupted capsular echo, strip echo, or sporadic echo in cracks.

CT diagnosis: A 64-layer spiral CT machine was used and operated under the following parameters: 130-150 mAs current, $120 \mathrm{kV}$ voltage, $50 \mathrm{~cm}$ view, $512 \times 512$ matrix, 2.5 mm layer thickness, 6-7 mm reestablished layer thickness, 1.25 screw pitch, and 150-250 HU window width. Scanning was performed from the hepatic dome to the symphysis pubis and lower right kidney. In clinics, the pelvic cavity can be scanned according to practical situations of patients. Retroperitoneum and intestinal injuries were examined carefully. Capsular hematoma was diagnosed by a stripped or crescent-shaped capsular membrane. Hematoma caused by organ damage was diagnosed by cracking or flake-like and stripped low-density shadow in organs.

\section{Observation index}

The two diagnostic methods were compared in terms of sensitivity, specificity, accuracy, and diagnosis accordance rate of organ (spleen, liver, kidney, pancreas, small intestine/ duodenum, and peritoneum) injury in the abdomen.

\section{Statistical analysis}

Data of the two diagnostic methods were analysed by SPSS22.0. Results were expressed in percentage and verified by $\chi^{2}$-test. Differences at $\mathrm{P}<0.05$ are not significant.

\section{Results}

\section{Surgical and pathological diagnostic results}

Seventy-five of the 80 patients recruited presented organ injury in the abdomen $(93.8 \%)$ and included 25 patients with spleen damage, 13 patients with liver damage, 10 patients with kidney damage, 10 patients with pancreas damage, 9 patients with small intestine/duodenum damage, and 8 patients with peritoneum damage.

\section{B-ultrasonography results}

The sensitivity, specificity, and accuracy of B-ultrasonography are $82.6 \%(62 / 75), 80.0 \% \quad(4 / 5)$, and $82.5 \% \quad(66 / 80)$, respectively (Table 1$)$.

Table 1. B-ultrasonography results.

$\begin{array}{lll}\begin{array}{l}\text { Surgical } \\ \text { pathological } \\ \text { diagnosis }\end{array} & \text { and } & \begin{array}{l}\text { B-ultrasonography } \\ \text { Organ injury in the No organ injury in } \\ \text { abdomen }\end{array}\end{array}$

\begin{tabular}{llcc}
\hline $\begin{array}{l}\text { Organ injury in the } 62 \\
\text { abdomen }\end{array}$ & 13 & 75 \\
\hline $\begin{array}{l}\text { No organ injury in the } \\
\text { abdomen }\end{array}$ & 4 & 5 \\
\hline Total & 63 & 17 & 80 \\
\hline
\end{tabular}

\section{CT diagnostic results}

The sensitivity, specificity, and accuracy of CT scan are $90.7 \%$ $(68 / 75), 80.0 \%(4 / 5)$, and 90.0\% (72/80), respectively (Table 2).

Table 2. CT diagnostic results.

\begin{tabular}{|c|c|c|c|}
\hline \multirow{2}{*}{$\begin{array}{l}\text { Surgical } \\
\text { pathological } \\
\text { diagnosis }\end{array}$} & \multicolumn{2}{|l|}{ CT diagnosis } & \multirow[t]{2}{*}{ Total } \\
\hline & $\begin{array}{l}\text { Organ injury in the } \\
\text { abdomen }\end{array}$ & $\begin{array}{l}\text { No organ injury in } \\
\text { the abdomen }\end{array}$ & \\
\hline $\begin{array}{l}\text { Organ injury in the } \\
\text { abdomen }\end{array}$ & 68 & 7 & 75 \\
\hline $\begin{array}{l}\text { No organ injury in the } \\
\text { abdomen }\end{array}$ & 1 & 4 & 5 \\
\hline Total & 69 & 11 & 80 \\
\hline
\end{tabular}

\section{Comparison between B-ultrasonography and CT diagnostic results}

The sensitivity, specificity, and accuracy of B-ultrasonography are $82.6 \%, 80.0 \%$, and $82.5 \%$, which are similar to those of CT scan $(90.7 \%, 80.0 \%$, and $90.0 \%$, respectively; $\mathrm{P}>0.05$; Table 3).

Table 3. Comparison between B-ultrasonography and CT scan (n $(\%))$.

\begin{tabular}{llll}
\hline $\begin{array}{l}\text { Diagnostic } \\
\text { method }\end{array}$ & $\begin{array}{l}\text { Sensitivity } \\
(\mathbf{n}=75)\end{array}$ & Specificity $(\mathbf{n}=5)$ & Accuracy $(\mathbf{n = 8 0})$ \\
\hline B-ultrasonography & $62(82.6)$ & $4(80.0)$ & $66(82.5)$ \\
\hline CT & $68(90.7)$ & $4(80.0)$ & $72(90.0)$ \\
\hline$X^{2}$ & 2.0769 & 0.0000 & 1.8972 \\
\hline$P$ & 0.1495 & 1.0000 & 0.1683 \\
\hline
\end{tabular}

\section{Diagnosis accordance rates of B-ultrasonography in organ injury in the abdomen}

The diagnosis accordance rates of B-ultrasonography in the injuries in the spleen, liver, kidney, pancreas, small intestine/ duodenum, and peritoneum are $92.0 \%, 92.3 \%, 100.0 \%, 80.0 \%$, $77.8 \%$, and $75.0 \%$, respectively (Table 4 ).

Table 4. Diagnosis accordance rates of B-ultrasonography in organ injury in the abdomen.

\begin{tabular}{llll}
\hline Organ injury in the abdomen & Surgical and pathological diagnosis & B-ultrasonography & Diagnosis accordance rate (\%) \\
\hline Spleen injury & 25 & 23 & 92.0 \\
\hline
\end{tabular}




\begin{tabular}{llll}
\hline Liver injury & 13 & 12 & 92.3 \\
\hline Kidney injury & 10 & 10 & 100.0 \\
\hline Pancreas injury & 10 & 8 & 80.0 \\
\hline Small intestine/duodenum injury & 9 & 7 & 77.8 \\
\hline Peritoneum injury & 8 & 6 & 75.0 \\
\hline
\end{tabular}

\section{Diagnosis accordance rates of CT scan in organ injury in the abdomen}

The diagnosis accordance rates of CT scan in the injuries to the spleen, liver, kidney, pancreas, small intestine/duodenum, and peritoneum are $100.0 \%, 100.0 \%, 90.0 \%, 90.0 \%, 100.0 \%$, and $87.5 \%$, respectively (Table 5).

Table 5. Diagnosis accordance rate of CT scan in organ injury in the abdomen.

\begin{tabular}{llll}
\hline $\begin{array}{l}\text { Organ injury } \\
\text { the abdomen }\end{array}$ & $\begin{array}{l}\text { Surgical and pathological } \\
\text { diagnosis }\end{array}$ & CT scan & $\begin{array}{l}\text { Diagnosis } \\
\text { accordance rate }\end{array}$ \\
\hline Spleen injury & 25 & 25 & 100.0 \\
\hline Liver injury & 13 & 13 & 100.0 \\
\hline Kidney injury & 10 & 9 & 90.0 \\
\hline Pancreas injury & 10 & 9 & 90.0 \\
\hline $\begin{array}{l}\text { Small intestine/ } \\
\text { duodenum injury }\end{array}$ & 9 & 9 & 100.0 \\
\hline Peritoneum injury & 8 & 7 & 87.5 \\
\hline
\end{tabular}

\section{Comparison between B-ultrasonography and CT scan in organ injury in the abdomen}

The diagnosis accordance rate of B-ultrasonography in the injuries to the spleen, liver, kidney, pancreas, small intestine/ duodenum, and peritoneum are 92.0\% (23/25), 92.3\% (12/13), $100.0 \%(10 / 10), 80.0 \%(8 / 10), 77.8 \%(7 / 9)$, and $75.0 \%(6 / 8)$, whereas those of CT scan are $100.0 \%(25 / 25), 100.0 \%(13 / 13)$, $90.0 \%(9 / 10), 90.0 \%(9 / 10), 100.0 \%(9 / 9)$, and $87.5 \%(7 / 8)$; the differences are not significant $\left(\chi^{2}=2.0833,1.0400,1.0526\right.$, $0.3921,2.2500,0.4102$. $\mathrm{P}=0.1489,0.3078,0.3049$, 0.5311, $0.1336,0.5218>0.05)$.

\section{Discussion}

Abdominal trauma is a common clinical traumatic injury and mainly includes open wounds and closed injuries. The diagnosis and treatment of abdominal trauma should emphasize on organ injury in the abdomen. Traumas on other parts of the body manifest slight clinical symptoms and physical signs and are non-life threatening [4]. By contrast, abdominal trauma is serious and often accompanied with nausea, emesis, stomachache, and hematuresis. The injury progresses gradually and may even lead to death. In clinics, appropriate treatment can save the life of patients successfully. Appropriate nursing care can effectively improve the prognosis of patients [5]. Timely and accurate diagnosis should be performed before any intervention is administered. Only with accurate diagnosis that patients be given appropriate intervention and nursing care, thereby ensuring the safety of patients and improving their prognosis.

B-ultrasonography and CT scan are widely used in clinical diagnosis. These procedures are simple, convenient, noninvasive, and accurate. In abdominal trauma diagnosis, imaging manifestations of organ injury in the abdomen mainly include the following [6,7]. (1) Spleen injury: Bultrasonography shows interrupted spleen membrane, heterogeneous echo in the parenchyma and adjacent organs, and spleen effusion; CT scan shows uneven parenchyma density and hematocele surrounding the spleen. (2) Liver injury: B-ultrasonography shows nonuniform intrahepatic echo, irregular shape of the liver parenchyma, low echo zone with ambiguous boundaries, and surrounding effusion; CT scan shows nonuniform liver density and surrounding hematocele. (3) Kidney injury: B-ultrasonography presents low echo zone and hematoma surrounding the kidney; CT scan presents nonuniform kidney density and surrounding hematocele. (4) Pancreas: B-ultrasonography shows nonuniform pancreas echo and surrounding effusion; CT scan shows nonuniform pancreas density and surrounding hematocele.

B-ultrasonography can be used to detect organ injuries and hematocele in the abdomen. This technique is simple to operate, low cost, and convenient to perform at bedside and thus has very high clinical application value [8]. The sensitivity, specificity, and accuracy of B-ultrasonography in abdominal trauma are very high; hence, this procedure can be applied and promoted in clinics. However, B-ultrasonography is sensitive to gases in the gastrointestinal tract, and this property influences the diagnosis accuracy [9]. CT scan exhibits high resolution and is insensitive to gases in the gastrointestinal tract. This method can detect organ injuries in the abdomen according to the density shadow in scanned positions. However, CT scan presents limitations in practical applications because of its high cost and inapplicability to patients with unstable hemodynamics [10]. Consistent with related literature, the present results demonstrate that in abdominal trauma diagnosis, B-ultrasonography and CT scan have no significant difference in term of sensitivity, specificity, accuracy, and diagnosis accordance rate of organ injury in the abdomen $(\mathrm{P}>0.05)$. Therefore, appropriate diagnosis methods should be selected according to the actual conditions of patients in clinics. 


\section{Conclusion}

B-ultrasonography and CT scan present similar results in abdominal trauma diagnosis. These techniques are noninvasive, simple to operate, and worthy of further clinical promotion and application.

\section{References}

1. Gong J, Mei D, Yang M, Xu J, Zhou Y. Emergency CT of blunt abdominal trauma: experience from a large urban hospital in Southern China. Quant Imaging Med Surg 2017; 7: 461-468.

2. Zhang Z, Li H, Meng X. A novel co (II)-based coordination polymer: anti-inflammatory activity in post-traumatic osteoarthritis. Lat Am J Pharm 2016; 35: 2284-2287.

3. Arbra CA, Vogel AM, Zhang J. Acute procedural interventions after pediatric blunt abdominal trauma: A prospective multicenter evaluation. J Trauma Acute Care Surg 2017; 83: 597-602.

4. Mahdi TN, Gholamhassan V, Hossein AE, Vida H. Acute and chronic effects of opiates and dopamine on yawning, penile erection and genital grooming behaviors in male Wistar rats. Biomed Res India 2017; 28: 6931-6936.

5. Echavarria Medina A, Morales Uribe CH, Echavarria RLG. Associated factors to non-operative management failure of hepatic and splenic lesions secondary to blunt abdominal trauma in children. Rev Chil Pediatr 2017; 88: 470-477.

6. Hafiz TA, Mubaraki MA. The potential role of Ziziphus spina-christi leaf extracts against Plasmodium bergheiinduced liver and spleen injury. Biomed Res India 2016; 27: 1027-1032.
7. Kloth C, Thaiss WM, Hetzel J, Bier G, Wirths S, Nikolaou $\mathrm{K}$, Horger M. Results of quantitative chest-CT in chronic pulmonary graft-vs.-host disease (cGvHD) 3 years after allogeneic stem cell transplantation. J Thorac Dis 2017; 9: 2521-2527.

8. Feng XR, Liang LH, Wu JF, Chen YQ, Liang S. 64-Slice spiral double-low CT to evaluate the degree of stenosis and plaque composition in diagnosing coronary artery disease. Exp Ther Med 2017; 14: 3088-3092.

9. Gausden EB, Nwachukwu BU, Schreiber JJ, Lorich DG, Lane JM. Opportunistic use of CT imaging for osteoporosis screening and bone density assessment: a qualitative systematic review. J Bone Joint Surg Am 2017; 99: 1580-1590.

10. Xiao W, Dai M. Formulation and characterization of biodegradable implants of ibuprofen for prolonged delivery in post-traumatic spinal injuries. Lat Am J Pharm 2015; 34: 1718-1722.

\section{*Correspondence to}

Xiang Li

Department of Radiology

Wuhan Central Hospital

Affiliated to Tongji Medical College of Huazhong University of Science and Technology

PR China 